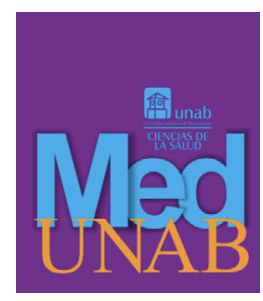

REVISTA DE LA FACULTAD

DE CIENCIAS DE LA SALUD

\title{
Uso y percepción del personal de salud sobre una aplicación móvil para la valoración geriátrica integral
}

Healthcare personnel use and perception of a mobile application for a comprehensive geriatric assessment.

Uso e percepção do pessoal de saúde de um aplicativo móvel para avaliação geriátrica ampla

Heider Alexis Bautista-Mier, MD. Esp. ${ }^{1}$ (D), Andrés Fernando Rodríguez-Gutiérrez MD. Esp. ${ }^{2}$ (D), Catalina Torres-Espinosa, MD. Esp. ${ }^{3}$ (D), Jorge Hernán López-Ramírez, MD. Esp. ${ }^{4}$ iD

1. Médico, Especialista en Geriatría. Universidad Nacional de Colombia, Bogotá, Colombia.

2. Médico, Especialista en Medicina Interna. Universidad Nacional de Colombia, Bogotá, Colombia.

3. Médico, Especialista en Geriatría. Docente de la Universidad Nacional de Colombia, Bogotá, Colombia.

4. Médico, Especialista en Geriatría. Docente de la Universidad Nacional de Colombia, Bogotá, Colombia.

Correspondencia. Heider Alexis Bautista Mier. Universidad Nacional de Colombia, Hospital Universitario Nacional de Colombia. Email. hbauta2@gmail.com - hbautistam@unal.edu.co

\section{INFORMACIÓN DEL ARTÍCULO:}

Artículo recibido: 22 de noviembre de 2020

Artículo aceptado: 24 de mayo de 2021

DOI: https://doi.org/10.29375/01237047.4041

Cómo citar. Bautista-Mier HA, Rodríguez-Gutiérrez AF, Torres-Espinosa C, López-Ramírez JH. Uso y percepción del personal de salud sobre una aplicación móvil para la valoración geriátrica integral. MedUNAB. 2021;24(2): 169-175. doi: https://doi.org/10329375/01237047.4041

\section{RESUMEN:}

Introducción. Existe poca evidencia que sustente la utilidad del uso de aplicaciones móviles en la atención del adulto mayor. GeriatriApp es una aplicación creada para dispositivos Android que busca facilitar al profesional de salud la realización de 
la valoración geriátrica integral (VGI) e integra la evaluación funcional, nutricional, cognitivo, emocional, farmacológico, fragilidad y sarcopenia. El objetivo del presente estudio es evaluar la utilidad percibida entre los usuarios de la App al momento de realizar la VGI, así como el efecto en la toma de decisiones y evaluar posibles diferencias entre el personal de salud. Metodología. Estudio descriptivo de corte trasversal, tipo encuesta entre usuarios de GeriatriApp, se indaga por aspectos demográficos, educativos y de percepción de utilidad de la App. Resultados. Se analizaron 228 encuestas. El 29\% fueron médicos especialistas, 18\% médicos generales, 17\% estudiantes de medicina y $12 \%$ médicos residentes. El grupo de edad con mayor frecuencia de uso de la App se encuentra entre 20 y 30 años (39\%). El principal escenario de práctica profesional fue el hospitalario (40.1\%), seguido de atención primaria (31.6\%). El 63\% utilizó la App entre 1 y 5 veces por semana. Durante el análisis estadístico no se encontró diferencia significativa en la frecuencia de uso según la edad ( $\mathrm{p}=0.631)$, ni el nivel de formación ( $\mathrm{p}=0.749)$. Para el 98\% de los encuestados la App facilitó la práctica clínica profesional y la toma de decisiones, y en el 99\% permitió identificar problemas en el adulto mayor en menor tiempo. Discusión. La aplicación estudiada tuvo un alcance internacional, lo que demuestra el potencial de este tipo de tecnologías. La mayor cantidad de usuarios fueron menores de 30 años, posiblemente explicado por su mayor contacto y experiencia con la tecnología móvil. No se encontraron diferencias en la frecuencia de uso según la edad, un hallazgo inesperado respecto a otros estudios. La percepción favorable de la aplicación parece estar secundada por su gratuidad, la utilidad de sus herramientas y una interfaz de uso sencilla. Conclusiones. GeriatriApp tiene una percepción favorable de utilidad entre el personal de la salud, permite realizar una valoración geriátrica en corto periodo de tiempo, facilita atención y toma de decisiones en el adulto mayor. Los resultados sugieren su utilidad en escenarios hospitalarios, atención primaria, centros de rehabilitación y hogares geriátricos.

Palabras clave:

Telemedicina; Salud Móvil; Aplicaciones Móviles; Adulto Mayor; Evaluación Geriátrica.

\section{ABSTRACT}

Introduction. There is little evidence supporting the usefulness of mobile applications in senior care. GeriatriApp is an application created for Android devices to assist health professionals in performing the comprehensive geriatric assessment (CGA). It integrates functional, nutritional, cognitive, emotional, pharmacological, frailty, and sarcopenia assessment. The aim of the present study is to evaluate the perceived usefulness of the App by its users when performing the CGA, as well as its effect on decision-making and to evaluate possible differences among healthcare personnel. Methodology. This survey-type descriptive cross-sectional study surveys GeriatriApp users, considering aspects like demographics, education, and the App's perceived usability. Results. A total of 228 surveys were analyzed, of which $29 \%$ involved specialist physicians, $18 \%$ general practitioners, $17 \%$ medical students, and $12 \%$ resident physicians. The age group with the highest frequency of App use was between 20 and 30 years old (39\%). The main professional practice setting was the hospital $(40.1 \%)$, followed by primary care $(31.6 \%)$. Sixty-three percent of the surveyed used the App between 1 and 5 times per week. The statistical analysis showed no significant difference in the frequency of use according to age $(\mathrm{p}=0.631)$ or education level $(\mathrm{p}=0.749)$. For $98 \%$ of the respondents, the App facilitated professional clinical practice and decision making, and, for $99 \%$, it allowed identifying problems in the seniors in less time. Discussion. The application studied has an international scope, demonstrating this technology's potential. The largest number of users were under 30, possibly because of their greater interaction and experience with mobile technology. No differences were found in the frequency of use according to age, an unexpected finding regarding other studies. The application's favorable perception seems to rely on the fact that it is free of charge, the tool's usability, and its user-friendly interface. Conclusions. GeriatriApp has a favorable perception of usefulness among health personnel, allows a geriatric assessment in a shorter period, and facilitates care and decision-making regarding seniors. The results suggest its usefulness in hospital settings, primary care, rehabilitation centers, and nursing homes.

Keywords:

Telemedicine; Mobile Health; Mobile Applications; Seniors; Geriatric Evaluation 


\section{RESUMO}

Introdução. Existem poucas evidências para apoiar a utilidade do uso de aplicativos móveis no atendimento dos idosos. GeriatriApp é um aplicativo criado para dispositivos Android que visa facilitar ao profissional de saúde a realização da avaliação geriátrica ampla (AGA) e integra a avaliação funcional, nutricional, cognitiva, emocional, farmacológica, fragilidade e sarcopenia. O objetivo deste estudo é avaliar a utilidade percebida pelos usuários do App no momento da realização da AGA, bem como o efeito na tomada de decisão, também avaliar possíveis diferenças entre os profissionais de saúde. Metodologia. Estudo transversal descritivo, tipo enquete entre usuários do GeriatriApp, consultando aspectos demográficos, educacionais e de percepção de utilidade do App. Resultados. Foi analisado um total de 228 enquetes. $29 \%$ eram médicos especialistas, $18 \%$ clínicos gerais, $17 \%$ estudantes de medicina, e $12 \%$ residentes. O grupo etário com maior frequência de utilização do $A p p$ tem entre 20 e 30 anos (39\%). O principal cenário de prática profissional foi o hospital (40.1\%), seguido da atenção básica (31.6\%). De outro lado, $63 \%$ usaram o aplicativo entre 1 e 5 vezes por semana. Durante a análise estatística, não foi encontrada diferença significativa na frequência de uso em função da idade $(p=0.631)$, nem do nível de educação $(p=0.749)$. Para $98 \%$ dos entrevistados, o App facilitou a prática clínica profissional e a tomada de decisões, e para $99 \%$ permitiu identificar problemas nos idosos em menos tempo. Discussão. $\mathrm{O}$ aplicativo estudado teve um alcance internacional, o que mostra o potencial desse tipo de tecnologia. O maior número de usuários tinha menos de 30 anos, possivelmente explicado pelo maior contato e experiência com a tecnologia móvel. Não foram encontradas diferenças na frequência de uso de acordo com a idade, uma descoberta inesperada em comparação com outros estudos. A percepção favorável do aplicativo parece apoiar-se na sua gratuidade, na utilidade das suas ferramentas e numa interface amigável. Conclusões. O GeriatriApp tem uma percepção favorável de utilidade entre os profissionais de saúde, permite uma avaliação geriátrica em um curto período de tempo, facilita a tomada de decisão e o atendimento ao idoso. Os resultados sugerem sua utilidade em ambientes hospitalares, atenção básica, centros de reabilitação e lares geriátricos.

Palavras-chave:

Telemedicina; Saúde Móvel; Aplicativos móveis; Idoso; Avaliação Geriátrica

\section{Introducción}

La mHealth (Mobile Health, por su acrónimo en inglés) o Salud Móvil, en español, es un concepto que propone la utilización de las tecnologías de información y las comunicaciones en dispositivos móviles con el objetivo de prevenir, diagnosticar y tratar la enfermedad o promoción de la salud (1). Su uso por profesionales de la salud facilita el acceso a la información, mejora la atención y la toma de decisiones y reduce el riesgo de error médico $(2,3)$. Cada año se solidifica la evidencia disponible del efecto positivo de la $m$ Health en el cuidado de la salud y su costo-efectividad $(4,5)$.

En geriatría las aplicaciones móviles (Apps) se utilizan en el manejo de condiciones crónicas, estimulación cognitiva, rehabilitación y actividad física $(6,7)$; pocas están enfocadas en la valoración geriátrica integral(VGI), a pesar de su beneficio en reducción de hospitalizaciones, institucionalización, discapacidad, y mortalidad $(8,9)$. Autores como Silva y colaboradores han identificado esta debilidad y han desarrollado GeriatricHelper (10). Aislan Lander desarrolla escalas geriátricas (11); sin embargo, se encuentran disponible en portugués. Otras Apps como Indicadores de dependencia (12), Valoración de la Fragilidad (13), aunque se encuentran en castellano, tienen la limitante que no permiten evaluar los diferentes dominios de la VGI.

GeriatriApp es una aplicación para tabletas y teléfonos inteligentes desarrollada por la unidad de Geriatría de la Universidad Nacional de Colombia. Está disponible de forma gratuita para el sistema operativo Android distribuida a través de la tienda de aplicaciones "Google Play", disponible para descarga en todo el mundo. GeriatriApp es para trabajadores de la salud que atienden la población adulta mayor y tiene como objetivo facilitar la VGI mediante la entrega de información de interés, siendo su principal utilidad el presentar escalas validadas para la evaluación funcional, estado nutricional, cognitivo, emocional, farmacológico, médico y de fragilidad $(14,15)$.

El objetivo del presente estudio es describir el perfil demográfico y educativo de los usuarios de GeriatriApp, así como el patrón de uso y su utilidad percibida entre 
estos al momento de realizar la VGI; incluyendo el posible efecto en la toma de decisiones en los diferentes escenarios de la práctica clínica. Así mismo, explorar posibles diferencias en el perfil de uso según la edad, el nivel de formación académica, el ámbito de práctica y el país de uso.

\section{Metodología}

Estudio descriptivo de corte transversal, en el cual se aplicó una encuesta anónima, de libre acceso a través de un formulario asequible desde la interfaz principal de GeriatriApp entre 2019 y 2020, con muestreo no probabilístico de tipo consecutivo. La encuesta fue diseñada a través de la plataforma de formularios Google; la información obtenida fue almacenada en hojas de cálculo. Las variables de estudio fueron datos demográficos como el país donde realiza la práctica profesional, el género, la edad del profesional, el nivel de formación académica y el escenario donde se realiza la atención. Se indagó si la aplicación facilitaba la práctica profesional, la toma de decisiones, si hace más rápida la valoración geriátrica y el número de veces por semana que utiliza la aplicación.

Se comparó la frecuencia de uso de la aplicación con la edad y el tipo de usuario de la App, y se evaluó la presencia de diferencias entre los países con mayor frecuencia de uso y el escenario de práctica.
Se incluyeron encuestas realizadas por el personal de salud con ejercicio en la atención del adulto mayor, con un uso de dos o más veces de la App. Se excluyeron encuestas con menos del $25 \%$ de los ítems resueltos o no aportar datos sobre ser trabajador de la salud. La invitación a realizar la encuesta se realizó directamente desde la App a todos los usuarios.

Para el análisis estadístico se tuvo en cuenta el tipo y la naturaleza de las variables; todas fueron cualitativas y se describieron con frecuencias absolutas y relativas. Para las pruebas de hipótesis se usó la prueba de independencia con Chi cuadrado sin corrección de Yates. Se consideró un error alfa de 0.05 para establecer significancia. Para el análisis estadístico se utilizó el software STATA 16.1.

\section{Resultados}

Se obtuvieron 235 encuestas, 7 fueron excluidas por datos incompletos. Los cinco países con mayor frecuencia de respuesta fueron Colombia $(38.2 \%)$, México $(18 \%)$, Argentina (13.16\%), España (6.6\%) y Chile (6.14\%). La proporción de hombres fue del $59.9 \%$. El grupo de edad con mayor frecuencia de uso de la App se encuentra entre 20 y 30 años, que corresponde al $39 \%$ de los encuestados, siendo de proporción aún mayor entre mujeres $(46.9 \%)$ (Tabla 1). Se halló una diferencia significativa en la distribución de edad entre hombres y mujeres $(\mathrm{p}<0.001)$.

Tabla 1. Distribución por edad y sexo de los encuestados

\begin{tabular}{|c|c|c|c|}
\hline Grupo de edad & Masculino & Femenino & Total \\
\hline & $\begin{array}{c}\text { Frec. abs. } \\
{[\text { Frec. rel. fila] }} \\
\text { [Frec. rel. columna] }\end{array}$ & $\begin{array}{c}\text { Frec. abs. } \\
\text { [Frec. rel. fila] } \\
\text { [Frec. rel. columna] }\end{array}$ & $\begin{array}{c}\text { Frec. abs. } \\
{[\text { Frec. rel. fila] }} \\
{[\text { Frec. rel. columna }]}\end{array}$ \\
\hline 20 a 30 años & $\begin{array}{c}44 \\
{[49.4 \%]} \\
{[33.3 \%]}\end{array}$ & $\begin{array}{c}45 \\
{[50.6 \%]} \\
{[46.9 \%]}\end{array}$ & $\begin{array}{c}89 \\
{[100 \%]} \\
{[39.0 \%]}\end{array}$ \\
\hline 31 a 40 años & $\begin{array}{c}32 \\
{[62.8 \%]} \\
{[24.2 \%]}\end{array}$ & $\begin{array}{c}19 \\
{[37.2 \%]} \\
{[19.8 \%]}\end{array}$ & $\begin{array}{c}51 \\
{[100 \%]} \\
{[22.4 \%]}\end{array}$ \\
\hline 41 a 50 años & $\begin{array}{c}18 \\
{[40.0 \%]} \\
{[13.6 \%]}\end{array}$ & $\begin{array}{c}27 \\
{[60.0 \%]} \\
{[28.1 \%]}\end{array}$ & $\begin{array}{c}45 \\
{[100 \%]} \\
{[19.7 \%]}\end{array}$ \\
\hline Mayor de 50 años & $\begin{array}{c}38 \\
{[88.4 \%]} \\
{[28.8 \%]}\end{array}$ & $\begin{array}{c}5 \\
{[11.6 \%]} \\
{[3.2 \%]}\end{array}$ & $\begin{array}{c}43 \\
{[100 \%]} \\
{[18.9 \%]}\end{array}$ \\
\hline Total & $\begin{array}{c}132 \\
{[57.9 \%]} \\
{[100.0 \%]}\end{array}$ & $\begin{array}{c}96 \\
{[42.1 \%]} \\
{[100.0 \%]}\end{array}$ & $\begin{array}{c}228 \\
{[100 \%]} \\
{[100 \%]}\end{array}$ \\
\hline
\end{tabular}

Abs: Absoluto. Frec: frecuencia. Rel: relativa.

Fuente: elaboración propia. 
El escenario de práctica donde se utiliza GeriatriApp con mayor frecuencia son el hospital $(40.1 \%)$, atención primaria $(31.6 \%)$, centros de rehabilitación $(9.6 \%)$ y en hogares geriátricos (6.1\%) (Tabla 2). En esta variable no hubo desbalance en la distribución según el sexo $(\mathrm{p}=0.646)$. Respecto al grado formativo, los usuarios más frecuentes de la App fueron médicos especialistas $(28.95 \%)$, médicos generales $(18 \%)$, estudiantes de medicina $(17.1 \%)$, residentes $(12.3 \%)$ y otras ocupaciones afines a la medicina (11.8\%) (Tabla 3 ).

Tabla 2. Frecuencia uso de la aplicación según el escenario de práctica, nivel formativo

\begin{tabular}{|c|c|c|c|}
\hline & Frecuencia absoluta & Frecuencia relativa & Frecuencia relativa acumulada \\
\hline Hospital & 92 & $40.4 \%$ & $40.4 \%$ \\
\hline Atención primaria & 72 & $31.6 \%$ & $71.9 \%$ \\
\hline Centro de rehabilitación & 22 & $9.6 \%$ & $81.6 \%$ \\
\hline Hogar geriátrico & 14 & $6.1 \%$ & $87.7 \%$ \\
\hline Urgencias & 13 & $5.7 \%$ & $93.4 \%$ \\
\hline Atención domiciliaria & 12 & $5.3 \%$ & $98.7 \%$ \\
\hline Otros & 2 & $0.9 \%$ & $99.6 \%$ \\
\hline UCI & 1 & $0.4 \%$ & $100 \%$ \\
\hline Total & 228 & $100 \%$ & -- \\
\hline
\end{tabular}

UCI: Unidad de Cuidados Intensivos.

Fuente: elaborado por los autores.

Tabla 3. Frecuencia de uso de la aplicación según el nivel formativo

\begin{tabular}{cccc} 
& Frecuencia absoluta & Frecuencia relativa & Frecuencia relativa acumulada \\
\hline Especialidad médica & 66 & $28.9 \%$ & $28.9 \%$ \\
\hline Médico general & 41 & $18.0 \%$ & $46.9 \%$ \\
\hline Pregrado en medicina & 39 & $17.1 \%$ & $64.0 \%$ \\
\hline Residencia médica & 28 & $12.3 \%$ & $76.3 \%$ \\
\hline $\begin{array}{c}\text { Ciencias afines a la } \\
\text { medicina }\end{array}$ & 27 & $11.8 \%$ & $88.1 \%$ \\
\hline $\begin{array}{c}\text { Enfermería o auxiliar de } \\
\text { enfermería }\end{array}$ & 13 & $5.7 \% \%$ & $93.8 \%$ \\
\hline $\begin{array}{c}\text { Estudiante de carreras } \\
\text { afines a la medicina }\end{array}$ & 10 & $4.4 \%$ & $98.2 \%$ \\
\hline$\quad$ Otros & 4 & $1.8 \%$ & $100 \%$ \\
\hline Total & 228 & $100.0 \%$ & -- \\
\hline
\end{tabular}

Fuente: elaborado por los autores.

Después de evaluar el número de veces por semana que se utiliza la aplicación se documentó que el $63 \%$ de los usuarios la utilizan entre 1 y 5 veces, $22.5 \%$ la utiliza entre 5 y 10 veces, y el $14.5 \%$ más de 10 veces por semana. Al indagar sobre si la aplicación facilitó la práctica clínica, el 98.7\% de los encuestados indicó que sí; de forma similar, el $97.8 \%$ indicó que sí facilitó la toma de decisiones. Así mismo, el 99.7\% indicó que sí permitió identificar problemas en el anciano, y el 99.6\% afirmó que aumentaba la velocidad de la evaluación geriátrica.

Dado que la edad podría determinar la frecuencia de uso se evaluó si existía desbalance de las proporciones según los grupos de edad, sin encontrarlo $(\mathrm{p}=0.631)$. Tampoco se encontró diferencias significativas de la frecuencia de uso según el nivel de formación $(\mathrm{p}=0.749)$. 
Se encontraron diferencias entre los siguientes países y el escenario de práctica clínica (Figura 1). Al comparar Colombia con Argentina, la proporción de usuarios de la aplicación que lo usan en el escenario hospitalario es significativamente mayor en Colombia $(62.1 \%$ versus $26.7 \%, \mathrm{p}=0.013)$. El mismo fenómeno se observó al comparar Colombia y México (62.1\% versus $24.4 \%$, p $<0.001)$. Al contrastar a México con Argentina no se encontraron diferencias significativas entre uso según el ámbito de atención $(\mathrm{p}=0.181)$.

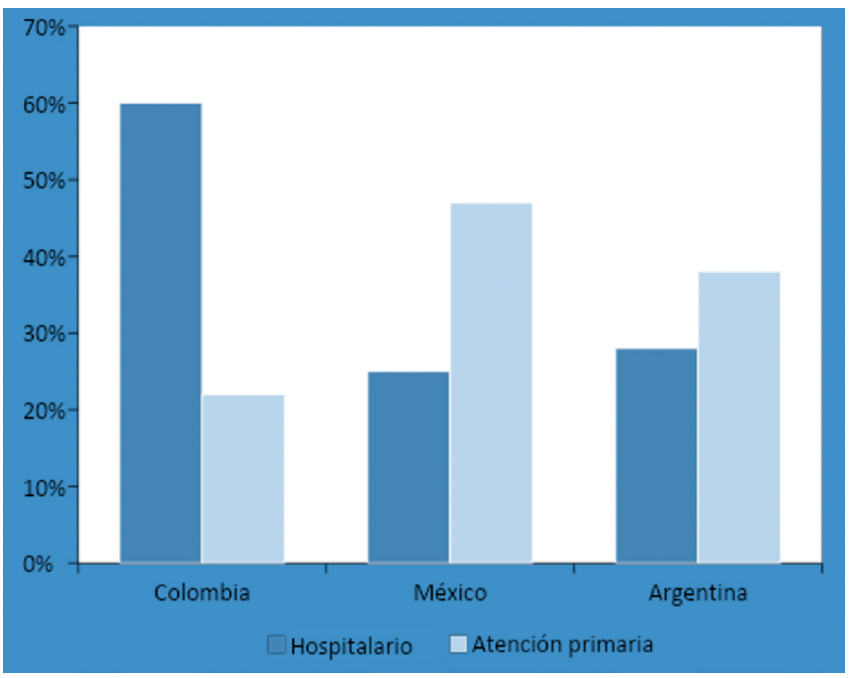

En Colombia, el $60 \%$ de los encuestados realizan su práctica en hospitalización y $22 \%$ en atención primaria. En México, el $25 \%$ en hospitalización y $47 \%$ en atención primaria. En Argentina, el $28 \%$ en hospitalización y el $38 \%$ en atención primaria.

Figura 1. Distribución del escenario de práctica entre los países con mayor número de encuestas realizadas.

Fuente: elaborado por los autores.

\section{Discusión}

GeriatriApp es una de las pocas aplicaciones en español diseñadas con el propósito de mejorar la accesibilidad de la VGI. El alcance de la App ha sido en toda Latinoamérica y España, siendo el país de origen (Colombia) el lugar de mayor uso (38.2\%); lo anterior es posiblemente explicado por la mayor difusión a través de diversos medios hechos desde el grupo creador.

La distribución de edad mostró mayor proporción de usuarios menores de 30 años, esto era esperable por ser una generación más expuesta y familiarizada con la tecnología de dispositivos móviles y a las aplicaciones, fenómeno similar observado en otros estudios. Sin embargo, es llamativo que, en el grupo de hombres, el segundo grupo con mayor frecuencia de uso fueron los mayores de 50 años. Es posible que este hallazgo sea una distorsión por un sesgo de quienes hicieron la encuesta, siendo un fenómeno a explorar en futuros estudios.

Los resultados arrojados en la encuesta no encontraron diferencias estadísticamente significativas en la frecuencia de uso de GeriatriApp entre los diferentes grupos etarios, ni diferencia según tipo de nivel formativo, lo cual no es concordante con estudios previos. Franko y colaboradores documentaron el menor uso de Apps médicas en aquellos con mayor experiencia (16). Payne y colaboradores realizaron una encuesta a estudiantes de medicina y médicos "juniors", encontraron un alto nivel de uso de apps en su práctica asistencial (17).

La percepción de utilidad y eficacia es alta entre los usuarios de la aplicación. La mayoría de los encuestados indicó que facilitó la práctica clínica y la identificación de problemas del paciente geriátrico, agilizando la VGI. Aunque se han descrito limitantes para el uso de Apps, como los costos, la utilidad de las herramientas y la usabilidad (18), los datos de esta encuesta sugieren que GeriatriApp supera estas tres barreras, posiblemente por su gratuidad, la amplitud de las utilidades que tiene al proponer las escalas de más frecuencia de uso en la mayoría de los dominios de la VGI, además de un diseño minimalista y sencillo.

Las limitaciones de este estudio surgen principalmente por la metodología de muestreo (no probabilístico de tipo consecutivo). Así mismo, no se cuantificó la proporción de no respuesta no se indagó las causas de quienes no respondieron; una hipótesis plausible es que los menos satisfechos con la aplicación la hayan desinstalado antes de responder, lo cual pudo sesgar las respuestas a los más satisfechos. Adicionalmente, al ser un estudio que evalúa la percepción de utilidad de la aplicación se desarrolló una herramienta de medición de novo sin seguir un proceso estandarizado para esto; lo anterior pudo haber limitado el entendimiento del fenómeno, pues pudieron dejarse de lado otros aspectos relevantes de la percepción y experiencia del usuario.

\section{Conclusiones}

GeriatriApp es percibida como una herramienta de gran utilidad para el personal de la salud, permite realizar la valoración geriátrica integral en menor tiempo, facilita la atención y toma de decisiones en los principales escenarios donde recibe los cuidados y atención en salud el adulto mayor. Es necesario profundizar más en el estudio de la mHealth en Latinoamérica, tanto en aspectos descriptivos en cuanto al uso y la experiencia del usuario, como también en el impacto real en desenlaces clínicos de este tipo de herramientas. 


\section{Financiación y conflictos de interés}

La presente investigación no ha recibido ayudas específicas provenientes de agencias del sector público, sector comercial o entidades sin ánimo de lucro. Los autores manifiestan ser los creadores y poseedores de los derechos de autoría de la aplicación, aclarando que la misma no genera ingresos por su carácter gratuito.

\section{Referencias}

1. Ventola CL. Mobile devices and apps for health care professionals: uses and benefits. P T [Internet]. 2014;39(5):356-64. Disponible en: https://www. ncbi.nlm.nih.gov/pmc/articles/PMC4029126/

2. Cortez NG, Cohen IG, Kesselheim AS. FDA regulation of mobile health technologies. N Engl J Med [Internet]. 2014;371(4):372-9. https://doi. org/10.1056/NEJMhle1403384

3. Chow CK, Ariyarathna N, Islam SMS, Thiagalingam A, Redfern J. mHealth in cardiovascular health care. Heart Lung Circ [Internet]. 2016;25(8):802-7. https:// doi.org/10.1016/j.hlc.2016.04.009

4. Elbert NJ, van Os-Medendorp $\mathrm{H}$, van Renselaar W, Ekeland AG, Hakkaart-van Roijen L, Raat H, et al. Effectiveness and cost-effectiveness of ehealth interventions in somatic diseases: a systematic review of systematic reviews and meta-analyses. J Med Internet Res [Internet]. 2014;16(4):e110. https://doi. org/10.2196/jmir.2790

5. Kitsiou S, Paré G, Jaana M, Gerber B. Effectiveness of mHealth interventions for patients with diabetes: An overview of systematic reviews. PLoS One [Internet]. 2017;12(3):e0173160. https://doi. org/10.1371/journal.pone. 0173160

6. Changizi M, Kaveh MH. Effectiveness of the mHealth technology in improvement of healthy behaviors in an elderly population-a systematic review. MHealth [Internet]. 2017;3:51 https://doi.org/10.21037/ mhealth.2017.08.06

7. Klimova B, Valis M. Smartphone applications can serve as effective cognitive training tools in healthy aging. Front Aging Neurosci [Internet]. 2017;9:436. https://doi.org/10.3389/fnagi.2017.00436

8. Stuck AE, Siu AL, Wieland GD, Rubenstein LZ, Adams J. Comprehensive geriatric assessment: a meta-analysis of controlled trials. Lancet [Internet]. 1993;342(8878):1032-6. https://doi. org/10.1016/0140-6736(93)92884-V

9. Ellis G, Whitehead MA, Robinson D, O'Neill D, Langhorne P. Comprehensive geriatric assessment for older adults admitted to hospital: meta-analysis of randomised controlled trials. BMJ [Internet]. 2011;343(oct27):d6553 https://doi.org/10.1136/bmj. d6553

10. Silva S, Felgueiras R, Oliveira IC. Geriatric Helper: An mHealth Application to Support Comprehensive Geriatric Assessment. Sensors (Basel) [Internet]. 2018 Apr 22;18(4):1285. https://doi.org/10.3390/ s18041285

11. Escalas Geriátricas [Internet]. Google.com. [citado el 20 de marzo de 2021]. Disponible en: https://play. google.com/store/apps/details?id=com.wecando. geriatria\&hl=es UY

12. Grupo Trevenque. Índices Dependencia. [Citado el 3 Mar 2021]. Disponible en: https://play. google.com/store/apps/details?id=gr.trevenque. indicadoresdependencia

13. Blue Bliss. Valoración de la fragilidad. [Internet]. [Citado 3 Mar 2021] Disponible en: https:// play.google.com/store/apps/details? $\mathrm{id}=\mathrm{com}$. escalasdefragilidad\&hl $=$ es_CO\&gl=US

14. Perafán Gaona DE, Bautista Mier HA. Construcción de una aplicación móvil basada en escalas de valoración geriátrica para profesionales de la salud. [Tesis de Especialización en Internet]. Bogotá, Colombia: Universidad Nacional de Colombia; 2018 [citado en marzo de 2021]. Disponible en: https://repositorio.unal.edu.co/ bitstream/handle/unal/69408/1130616200.2018. pdf? sequence $=1$ \&isAllowed $=y$

15. Bautista HA, Perafán D, Torres C, López JH. Presentación de GeriatriApp: una app para la evaluación geriátrica integral en español. Aten Primaria [Internet]. 2020;52(6):436-8. https://doi. org/10.1016/j.aprim.2019.07.011

16. Franko OI, Tirrell TF. Smartphone app use among medical providers in ACGME training programs. $\mathrm{J}$ Med Syst [Internet]. 2012;36(5):3135-9. https://doi. org/10.1007/s10916-011-9798-7

17. Payne KFB, Wharrad H, Watts K. Smartphone and medical related App use among medical students and junior doctors in the United Kingdom (UK): a regional survey. BMC Med Inform Decis Mak [Internet]. 2012;12(1):12117. https://doi.org/10.1186/1472-6947$12-121$

18. Gagnon M-P, Desmartis M, Labrecque M, Car J, Pagliari C, Pluye P, et al. Systematic review of factors influencing the adoption of information and communication technologies by healthcare professionals. J Med Syst [Internet]. 2012;36(1):24177. https://doi.org/10.1007/s10916-010-9473-4 